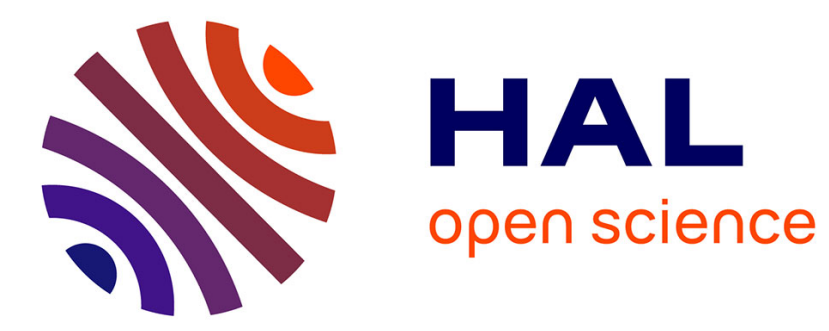

\title{
Citoyenneté et racisme en Europe : exception et complémentarité britanniques
}

\author{
Catherine Neveu
}

\section{To cite this version:}

Catherine Neveu. Citoyenneté et racisme en Europe: exception et complémentarité britanniques. Revue Europeenne des Migrations Internationales, 1994, 10 (1), pp.95-107. hal-00178145

\section{HAL Id: hal-00178145 \\ https://hal.science/hal-00178145}

Submitted on 10 Oct 2007

HAL is a multi-disciplinary open access archive for the deposit and dissemination of scientific research documents, whether they are published or not. The documents may come from teaching and research institutions in France or abroad, or from public or private research centers.
L'archive ouverte pluridisciplinaire HAL, est destinée au dépôt et à la diffusion de documents scientifiques de niveau recherche, publiés ou non, émanant des établissements d'enseignement et de recherche français ou étrangers, des laboratoires publics ou privés. 


\title{
Citoyenneté et racisme en Europe : exception et complémentarité britanniques
}

\author{
Catherine NEVEU
}

En 1986, le Parlement Européen émettait l'idée de mettre sur pied une structure au niveau européen, qui permette aux migrants "d'avoir leur voix à eux, parce qu'ils n'ont pas de voix politique" 1 . La volonté du Parlement Européen était alors de constituer un lieu d'expression pour les populations non-communautaires établies en Europe, au travers duquel elles puissent non seulement faire part de leurs revendications, mais également diffuser l'information émanant des instances européennes. Le processus devait donc être double : fournir aux instances européennes une source d'informations et de débats, tout en permettant une meilleure circulation de l'information en direction de ces populations. Ce souci paraissait d'autant plus répondre à une nécessité que certaines études avaient montré le faible niveau de connaissances quant aux lieux ou aux processus de décision dans les instances européennes parmi les associations de migrants ${ }^{2}$.

L'objectif de cet article n'est pas tant de considérer le fonctionnement du Forum des Migrants que d'examiner les difficultés auxquelles se confronte ce type d'initiatives en termes de catégories : quels doivent être les critères d'inclusion et d'exclusion dans ce Forum, existe-t-il des catégories qui soient pertinentes pour l'ensemble des pays européens ? En élargissant la réflexion à une autre structure à vocation européenne, SCORE (UK) ${ }^{3}$, les questions ouvertes par ces approches européennes, et/ou quelquesunes de leurs limites, pourront être abordées de manière dynamique.

\section{Le Forum des Migrants, pour qui ?}

1. Entretien avec M. Kendall, Commission des Communautés Européennes, Bruxelles, juin 1991.

2. Voir De Jong, D. et Zwamborn, M., 1991.

3. Standing Conference on Racial Equality in Europe. 
L'objectif défini pour le Forum des Migrants par le Parlement Européen étant de fournir aux populations qui n'en disposaient pas un espace d'expression politique, il fut d'emblée décidé d'exclure de cette structure les associations de résidents communautaires établis dans d'autres pays européens ; c'est ainsi que les associations italiennes en France ne furent pas invitées à participer à la réunion fondatrice du Forum. En effet, les autorités européennes estimaient que les populations grecques, italiennes, portugaises ou encore espagnoles établies dans d'autres pays de la Communauté que le leur disposaient, de par leur statut de ressortissants communautaires, de modes d'expression politique suffisants. Leurs membres peuvent participer aux élections européennes, ainsi qu'aux différentes structures existantes, et donc faire entendre leurs voix. Les canaux politiques "de droit commun" existant ont alors été considérés comme suffisants pour que les populations émigrées communautaires puissent influer sur les décisions prises au niveau européen.

On peut donc poser comme première constatation que la Commission des Communautés, à la demande insistante des parlementaires européens, a voulu mettre sur pied une structure s'adressant à ceux que Thomas Hammar appelle les denizens, c'est à dire des populations installées de manière stable et durable dans un pays européen, y jouissant d'un statut légal également stable, mais n'ayant pas pleinement accès aux modes d'expression politique ${ }^{4}$.

A première vue, le Forum des Migrants serait alors une structure dont le critère principal d'accès serait le statut : les citoyens communautaires ayant accès à la scène politique européenne de par leur citoyenneté nationale, seuls seraient représentées dans le Forum les populations immigrées extra-communautaires.

Des critères discutés

Si ce critère d'accès était cohérent avec la volonté du Parlement Européen, il ne va pas sans poser de problèmes à différents niveaux, et les critiques ne tardèrent pas à fuser.

La première porta sur la division ainsi introduite entre immigrés communautaires et extra-communautaires ; ainsi selon A. Perotti : "Il y a tout de suite eu des réactions de la part des ressortissants communautaires, des associations communautaires, qui ont mal vécu cet organisme qui risquait de rompre un petit peu l'unité de la communauté immigrée en général en Europe, en créant certaines frontières, certaines barrières" 5 . Il s'agit certes là d'une question incontournable dans la mesure où toute structure de ce type doit poser ses critères d'inclusion et d'exclusion; et s'il semble que certaines

4. T. Hammar.

5. Entretien avec A. Perotti, directeur du CIEMI, Paris, juin 1991. 
associations communautaires aient mal réagi à leur exclusion du Forum, celle-ci n'a apparemment pas soulevé les mêmes débats au sein des associations d'immigrés extracommunautaires.

Mais au-delà de la stricte question d'un accès à la parole pour des populations ne disposant pas de l'ensemble des droits politiques au niveau européen, il semble qu'un autre souci, une autre conception, aient présidé au choix même de ceux qui, parmi les associations d'immigrés extra-communautaires, allaient être inclus dans le Forum des Migrants. En effet, un des critères retenus par le CIEMI, chargé à l'époque de dresser une première liste des associations susceptibles de participer à sa mise en place, fut le rapport au pays, ou plus exactement à l'Etat d'origine. Toujours selon A. Perotti, il s'agissait d'éviter "que le Forum soit délégitimé aux yeux des pouvoirs politiques d'origine". Concrètement, ce dont il s'agissait était de signaler à la Commission des Communautés les organisations qui, tout en étant bien implantées en Europe, entretenaient des rapports sinon conflictuels, du moins critiques, avec les gouvernements d'origine ; l'exemple le plus immédiat de ce type d'organisations est bien sûr celui des associations kurdes, difficilement reconnues par le gouvernement turc.

Cette précaution partait certes d'une première idée pertinente, celle que les associations retenues devaient avoir pour objectifs principaux l'aménagement de la vie des migrants dans leur pays d'installation, et non les questions politiques dans leur pays d'origine.

Mais le souci de signaler les organisations n'entretenant pas des relations cordiales avec les gouvernements d'origine, et ce afin "de ne pas délégitimer le Forum" aux yeux de ceux-ci, paraît plus problématique. Tout d'abord parce que la conception à l'oeuvre ici induit l'idée que les populations concernées se situent encore principalement dans une configuration ayant pour centre le pays d'origine. S'il va de soi qu'un certain nombre de problèmes auxquels se confrontent les denizens extra-communautaires peuvent ou doivent se régler dans le cadre d'accords bi- ou multi-latéraux ${ }^{6}$, n'y a-t-il pas là un risque de voir se perpétuer un contrôle important des Etats d'origine sur leurs ressortissants émigrés, ce qui ne va pas sans soulever des questions importantes ? Plus encore, à partir de quand est-on fondé à considérer que telle ou telle organisation entretient de "bons rapports" avec son gouvernement d'origine ? Les critères sont-ils ceux fixés par ces mêmes gouvernements, sont-ils basés sur l'appréciation des associations elles-mêmes, ou encore sur une décision de la Commission des Communautés ? A titre d'exemple, l'Association des Marocains en France, constituée en partie comme riposte à la volonté du gouvernement marocain de contrôler ses

6. On peut penser par exemple aux questions de protection sociale ou d'accès à la nationalité. Voit T. Hammar. 
ressortissants par le biais de l'Amicale des Marocains, peut-elle être considérée comme oppositionnelle?

Enfin, s'il faut reconnaître que le Forum des Migrants a été mis en place dans le cadre de politiques d'Etats, même transcrites en instances transnationales, ne peut-on penser qu'il pouvait aussi fournir à ces populations une tribune leur permettant de faire pression sur leur propre gouvernement sur un certain nombre de questions, voire même d'énoncer des critiques sinon sur sa politique interne, du moins sur sa politique envers ses ressortissants émigrés. L'espace européen aurait alors pu là jouer également un rôle d'émancipation et de critiques à l'égard des gouvernements des pays d'origine.

La question qui reste donc ouverte ici est celle d'une première ambiguïté : le souci de faire du Forum des Migrants une structure légitime aux yeux des Etats d'origine n'est-il pas au moins partiellement contradictoire avec celui de donner aux denizens un espace d'expression; en maintenant ce critère, ne renvoie-t-on pas ces populations à un statut plus proche de celui traditionnel d'immigré, avec ce que cela comporte en termes des types de liens avec le pays d'origine, que de celui de denizen, dans lequel l'accent est mis surtout sur la durabilité et la stabilité du séjour en Europe ?

Une telle perception peut également laisser penser que le fait même de l'établissement prolongé dans un pays n'influe en aucune manière sur la perception qu'ont d'ellesmêmes les populations concernées; dans les processus dynamiques d'adaptation auxquels la migration, puis l'installation, donnent lieu, n'y a-t-il pas en effet modifications, évolutions des systèmes de références, qui ne peuvent plus être réduits ni à ceux de la société d'installation, ni à ceux de la société d'origine?

Mais l'initiative du Forum des Migrants pose encore d'autres questions. En poussant le trait, on pourrait ainsi se demander dans quelle mesure cette initiative n'est pas venue trop tard, dans quelle mesure elle n'est pas déjà en décalage par rapport à une partie de la réalité contemporaine.

En effet, en utilisant comme critère de base pour la participation à cette structure le fait de ne pas être citoyens d'un des pays de la Communauté Européenne, une fraction grandissante des populations issues de l'immigration en est exclus ; il s'agit notamment des jeunes, qui ont obtenu dans certains pays la nationalité et/ou l'accès aux droits politiques. A. Perotti est d'ailleurs conscient de ce problème : "Les gens des banlieues, est-ce qu'ils ont besoin de ça? Le Forum, c'est pour les anciens, c'est pas pour les jeunes. C'est une institution qui essaie de représenter les adultes de la première génération non-communautaires" 7.

7. Entretien avec A. Perotti, juin 1991. 
Une seconde division serait donc introduite, cette fois-ci au sein même des populations issues de l'immigration extra-communautaire, entre ceux qui ont accès aux droits politiques dans leur pays de résidence, et les autres. Il ne s'agit pas ici de nier la pertinence d'une structure telle que le Forum ; la nécessité d'un espace d'expression politique pour les populations à l'heure actuelle exclus des processus décisionnels est certaine, bien qu'on puisse s'interroger sur l'efficacité de cette initiative en l'absence de structures similaires au niveau des différents Etats membres. Ce dont il s'agit est de questionner sinon la pertinence, en tout cas la cohérence des critères mis en oeuvre.

En toute logique, le critère de la non-jouissance de la citoyenneté d'un des pays membres conduit à l'exclusion des associations de jeunes "beurs" en France, ceux-ci étant dans leur immense majorité des citoyens français ; de même pour les minorités ethniques en Grande-Bretagne, en tout cas pour celles ayant la nationalité britannique. Mais la "frontière" peut, en la matière, être encore plus floue ; ainsi l'Association des Marocains en France compte dans ses rangs un nombre croissant de Français d'origine marocaine. Cette situation pousse d'ailleurs cette association à s'interroger sur la pertinence de modifier son apppelation : "D'ailleurs, nous sommes obligés aujourd'hui de ne plus nous considérer comme "les Marocains", mais comme des communautés européennes, françaises, d'origine marocaine. /.../ Nous aurions pu l'appeler (1'AMF) maintenant l'Association des Marocains de France, ou des Marocains et originaires du Maroc de France" 8 . Il apparaît donc qu'y compris pour des associations de type plus "traditionnel", des évolutions importantes aient eu lieu depuis quelques années, rendant délicate une qualification homogène d'association de primo-migrants.

\section{L'exception britannique}

Mais il est temps maintenant d'en arriver à une des contradictions principales dans la définition des catégories concernées par le Forum des Migrants. Comme on l'a vu plus haut, il semble à première vue que le critère principal mis en oeuvre soit celui du statut : participent au Forum, par le biais de leurs associations, les migrants noncommunautaires.

Or une exception, et de taille, a été faite à ce principe, celle des Britanniques : "une exception d'ailleurs qu'on a faite, on avait dit que c'était pas pour les communautaires, parce que les communautaires ont déjà leur voix, comme les Grecs en Allemagne ou les Portugais au Luxembourg, ils ont déjà leur Parlement, parce qu'ils sont

8. Entretien avec M. Melhouk, AMF, Paris, juin 1991. 
communautaires. Il y a des cas, disons marginaux, ce qui veut dire les Britanniques" 9. Sans vouloir se prononcer sur la marginalité des Britanniques au sein de l'Europe, il convient toutefois de noter qu'il s'agit là d'une exception de taille, tant par le nombre des personnes concernées que par leur situation : ces Britanniques dont il est question ici ne sont pas, dans l'immense majorité des cas, des communautaires établis dans un autre pays de la Communauté, mais des citoyens à part entière résidant dans le pays même dont ils sont citoyens. L'exception britannique est donc double : sur le statut, puisque les populations concernées sont communautaires et votent à toutes les élections, y compris européennes ; et sur le lieu de résidence puisqu'il est celui-là même où ils sont citoyens.

Au nom de quoi alors cette exception a-t-elle été faite ? Laissons encore ici la parole à M. Kendall : "Il y a des cas marginaux, ce qui veut dire les Britanniques, bien qu'ils soient d'origine indienne, bangladeshie, pakistanaise, etc, ils ont la nationalité britannique. Mais ça ne veut pas dire que leurs problèmes sont terminés parce qu'ils ont le droit de vote et de libre circulation" 10

Plusieurs questions peuvent alors être posées. Si l'accès au Forum est basé sur le fait que "les problèmes ne soient pas terminés" malgré la jouissance des droits politiques, pourquoi en avoir exclus les autres populations communautaires, ou celles ayant la nationalité de leur pays d'installation? Les premières ne disposent généralement pas des droits politiques dans leur pays de résidence, et peuvent être victimes, tout comme les seconds d'ailleurs, de discriminations très réelles, comme le furent il y a quelques mois des Italiens établis en Allemagne. Il ne s'agit pas à l'évidence que de cela, puisque la possibilité qu'ont ces populations communautaires, et/ou celles jouissant de la nationalité du pays d'installation, d'élire des représentants dans les Parlements nationaux et européen paraît suffire à leur assurer une voix au chapitre.

L'inclusion des Britanniques est donc porteuse d'autre chose, qui n'a pas été nommé explicitement, à savoir la perpétuation d'inégalités de fait et de discriminations pour des citoyens originaires de pays tiers, généralement du Sud, ou pour utiliser le terme britannique, de "Black citizens". En incluant les minorités ethniques britanniques et en excluant les autres communautaires ainsi que les nationaux communautaires d'origine immigrée, le Conseil des Communautés Européennes n'a-t-il pas implicitement introduit un autre critère dans l'accès au Forum des Migrants, celui de la "race" ? Ce faisant, on peut penser qu'il s'est rendu aux importantes pressions d'associations britanniques, pour qui la lutte contre les discriminations raciales est au coeur des enjeux politiques et sociaux ; mais s' il y a eu là acceptation, même implicite, de la validité de leurs arguments, pourquoi n'avoir pas étendu la participation au Forum des Migrants à

9. Entretien avec M. Kendall, Commission des Communautés Européennes, Bruxxelles, juin 1991.

10. Idem. 
d'autres catégories de citoyens communautaires, qui sont eux aussi victimes de pratiques discriminatoires ?

Dès lors, la démarche même de cette structure semble entachée d'une ambiguïté de taille : son objectif est-il de regrouper des associations sur la base d'un statut, celui de denizen, pour leur donner un accès aux processus décisionnels ; ou s'agit-il de regrouper les populations victimes de discriminations raciales quelque soit leur statut? En d'autres termes, est-ce le statut ou l'origine "ethnique", "raciale" ou nationale qui fonde la légitimité du Forum ? Son objet est-il d'offrir une possibilité d'expression à des populations n'ayant pas accès aux canaux démocratiques des citoyens, ou d'être un Forum contre le racisme?

\section{Racisme ou citoyenneté : deux approches différentes ?}

Le Forum des Migrants fonctionne depuis bientôt trois ans, et dans l'attente d'études plus complètes sur son fonctionnement réel, on insistera surtout ici sur l'ambivalence plus globale que reflète cette structure, celle de savoir si dans l'espace européen d'après 1993, l'accent doit être mis sur la citoyenneté ou sur le racisme.

Ces deux approches ne sont pas mutuellement exclusives et la revendication d'une égalité des droits civils et politiques peut et doit s'accompagner de la lutte contre toutes les formes de discrimination. Elles mettent cependant en oeuvre deux logiques différentes, dont l'examen de quelques aspects de "l'exception britannique" peut révéler les contradictions principales. C'est afin d'éclairer les rapports entre ces deux démarches, de la lutte contre le racisme et de la citoyenneté, qu'une autre tentative de constitution d'un réseau européen pour les populations issues de l'immigration doit être examinée.

SCORE (UK) : le primat de la lutte contre les discriminations

La libre circulation au sein de l'Europe de 1993 paraît avec de plus en plus de force comme une des préoccupations majeures des associations, et des individus, issus de l'immigration, notamment avec la signature des accords de Schengen et du groupe Trevi. Cette Europe semble alors représenter tout à la fois un espoir et une crainte. L'espoir, c'est la remise en cause d'une certaine conception désignée comme "nationalitaire", qui permet d'entrevoir des possibilités d'accès à une citoyenneté 
européenne, y compris pour les résidents et les denizens non-communautaires. C'est ce qui est exprimé dans la revendication d'un European Travel Document, devant permettre la libre circulation, au même titre que les ressortissants des Etats membres, à tous les résidents, quelque soit leur nationalité.

Dans un certain nombre de cas, et le recours de plus en plus fréquent aux instances européennes contribue à l'indiquer, l'Europe est donc perçue comme une opportunité pour contourner et/ou dépasser des blocages ou des législations nationales. Ainsi, il semble que des associations en Belgique comptent beaucoup sur des pressions au niveau européen pour obtenir le droit de vote pour les non-nationaux, même si dans d'autres pays, comme l'Allemagne et le Danemark, les stratégies sont surtout nationales 11 .

Mais les craintes semblent souvent souvent plus centrales que les espoirs ; la principale inquiétude est alors de voir se mettre en place une liberté de circulation à deux vitesses, favorisant la mobilité des communautaires tout en restreignant fortement celle des résidents extra-communautaires, et à plus forte raison de tous ceux qui voudraient entrer dans une Europe devenue forteresse. La mise en place par les accords de Schengen d'un fichier unique dans lequel se retrouvent amalgamés trafiquants de drogues, grand bandistisme, terrorisme et "indésirables", cette dernière catégorie concernant notamment les réfugiés et tous ceux qui se seront vus refuser un visa dans l'un des pays signataires de l'accord, ne cesse d'inquiéter, avec raison, les associations travaillant avec des populations issues de l'immigration ou des réfugiés. L'absence quasi-totale de contrôle démocratique sur la mise en oeuvre d'accords de ce type, régis par les exécutifs des différents pays, rend l'inquiétude encore plus grande et justifiée quant à l'usage de ce genre de fichiers 12 .

Mais, et cela est particulièrement vrai en Grande-Bretagne, les craintes vis à vis de cette Europe-là ne s'expriment pas uniquement en termes de statut, mais aussi, voire surtout, en termes de racisme. C'est dans cette logique que s'est constituée dès 1990 une structure sinon concurrente, du moins parallèle, à celle du Forum des Migrants, SCORE (UK).

Le nom même de cette organisation (Standing Conference on Racial Equality) indique clairement que l'accent est mis là sur le racisme et les discriminations, et non sur le statut ; la mention UK quant à elle indique la volonté d'être la branche britannique d'une organisation qui couvrirait toute l'Europe. Les objectifs de SCORE (UK) sont "de travailler pour l'égalité de conditions de tous les résidents de la communauté

11. Voir De Jong, D. et Zwamborn, M., 1991, p. 25.

12. Sur ce sujet, voir en particulier le numéro spécial de Race and Class de janvier-mars 1991. 
européenne sans distinction de couleur, de race, de nationalité ou de religion; de promouvoir des actions et des législations pour obtenir un traitement égal pour les communautés black, migrantes ou autres minorités ethniques ; de promouvoir une législation qui rende le harcèlement racial, la violence contre les Blacks et autres personnes de couleur illégaux; et de contacter et coopérer afin de créer des réseaux de groupes et d'organisations à travers l'Europe pour l'échange d'informations" 13.

Le compte-rendu de la Conférence inaugurale de SCORE (UK) indique clairement les craintes quant aux obstacles réels mis à la libre circulation des "Blacks British people" :"LeTraité de Schengen a été signé par cinq pays parce que la Communauté n'était pas assez rapide dans sa prise en compte des dangers de la libre circulation des Blacks. Et "Black" signifie ici "immigrant". Claude Moraes, un Britannique, et donc un citoyen de la Communauté Européenne, est régulièrement désigné comme "un immigré"" 14.

L'argument est là très clair : les barrières mises à la libre circulation des migrants et des résidents influeront nécessairement sur celle des ressortissants communautaires "blacks", ces derniers étant systématiquement assimilés à des migrants.

Plusieurs des orateurs de cette Conférence insisteront d'ailleurs sur ce point, comme Bernie Grant, Membre du Parlement britannique et président de SCORE (UK), quand il dira qu'il y a "une idée fixe sur ce qu'est la citoyenneté européenne : white", les "gens de couleur" étant vus systématiquement comme des étrangers ${ }^{15}$. Les exemples sont en effet fréquent de ce type de discriminations aux frontières pour des citoyens blacks, systématiquement contrôlés, soupçonnés et dont la citoyenneté est par principe mise en doute par les polices des frontières. On retrouve d'ailleurs fréquemment le même sentiment désabusé chez de jeunes Français issus de l'immigration; l'accès à la nationalité et/ou à la citoyenneté ne fait pas miraculeusement disparaître les pratiques discriminatoires, et peut même parfois les renforcer, quand il est considéré que ce statut est usurpé.

L'analyse de cette situation proposée par SCORE (UK) a l'avantage de montrer à quel point racisme et perceptions de l'immigration sont aujourd'hui deux phénomènes qui, s'ils ne sont pas réductibles l'un à l'autre, entretiennent des liens étroits. Ainsi Anne

13. Plaquette de présentation de SCORE (UK).

14. SCORE (UK), 24/11/90, paragraphe 2.5.

15. Idem, paragraphe 1.4. Il est difficile de traduire des termes comme " white" et "black", car les connotations de leur transcription en français ne sont pas les mêmes ; c'est pourquoi on a préféré ici les conserver en anglais. 
Owers du JCWI 16 a soulevé, toujours lors de cette conférence inaugurale, la très réelle question des relations entre contrôle de l'immigration et égalité au sein des Etats : "Il y a eu un marchandage. La libre circulation des ressortissants (whites) communautaires se fait en échange d'un renforcement des contrôles tant internes qu'externes, qui affectent aussi bien les citoyens britanniques blacks que les non-citoyens. "Immigré" est un code pour "black" et il n'a jamais été possible d'avoir à la fois l'inégalité aux frontières et l'égalité en leur sein" 17.

Il s'agit là d'un point particulièrement important et pertinent. La désignation des migrants originaires des pays du Tiers-Monde comme une masse de population difficilement contrôlable, véritable menace pour l'Europe riche et à qui il serait légitime d'y refuser l'entrée, cette désignation ne peut pas se faire sans effets à l'intérieur, pour les populations issues de ces pays. Pourquoi un "indésirable" à la frontière serait-il plus désirable dans la cité, le quartier ou l'école ? D'autant plus que le développement des contrôles internes au nom de la lutte contre l'immigration clandestine vient encore réactiver l'amalgame entre black, immigré, clandestin et indésirable 18.

Comme le rappelle très justement Sivanandan: "La citoyenneté peut ouvrir les frontières européennes aux blacks et leur permettre de circuler librement, mais le racisme ne fait pas la différence entre un Black et un autre, un citoyen et un immigré, un immigré et un réfugié, et considère tous les gens du Tiers-Monde comme des immigrés et des réfugiés, et tous les immigrés et les réfugiés comme des terroristes et des trafiquants de drogue". C'est justement sur la base d'une telle analyse que SCORE (UK) s'est constitué : la lutte contre le racisme et pour l'égalité des chances serait au coeur des questions non pas soulevées, mais exacerbées par l'intégration européenne. Le statut n'est alors qu'un aspect subsidiaire d'un danger beaucoup plus grave, celui de la discrimination raciale pour tous les "gens de couleur" en Europe, qu'ils soient ou non ressortissants communautaires; et ce d'autant plus dans une situation d'exacerbation des discours et des pratiques légitimant une fermeture quasi-totale de l'Europe aux mouvements migratoires.

Les inquiétudes des Britanniques blacks sont d'autant plus grandes alors que l'Europe de 1993 paraît remettre en cause toute une série de droits et d'acquis conquis de haute lutte en Grande-Bretagne. Ce pays est en effet le seul en Europe à disposer d'une législation

16. Joint Council for the Welfare of Immigrants.

17. SCORE (UK), 24/11/90, paragraphe 5.3.

18. Ne peut-on penser d'ailleurs à ce sujet qu'il y a une contradiction importante entre la réflexion sur des politiques d'intégration au niveau européen d'une part, et le fait que les politiques d'immigration restent du ressort des Etats d'autre part? 
sur les "relations raciales", condamnant y compris les discriminations indirectes ; quelles que soient les critiques qui sont formulées à son égard par les organisations blacks elles-mêmes, cette législation n'en a pas moins le mérite d'exister,et donc d'inscrire sur l'agenda politique national la question de la lutte contre la discrimination raciale.

Il y a alors une crainte très sensible de voir l'intégration européenne entraîner un nivellement par le bas dans ce domaine. Cette crainte ne va d'ailleurs pas parfois sans un arrière-goût de "défense des avantages acquis" et de rejet de l'amalgame entre black et immigré, non pas dans une dimension positive, mais comme une comparaison péjorative pour les Blacks eux-mêmes 19.

Il est indéniable que la Grande-Bretagne fait figure d'exception sur la scène européenne en matière de "relations raciales", et le continent n'a d'ailleurs jamais été une référence pour les Britanniques blacks ; leur référence essentielle dans ce domaine reste les EtatsUnis, ce qui permet un double discours en ce qui concerne l'égalité des droits. Au niveau interne, ils insistent sur le fait que trop peu est fait en Grande-Bretagne ; vis à vis de l'Europe, ils estiment que ce pays est très largement en avance.

De façon plus positive donc, la volonté sous-tendant l'initiative de SCORE (UK) serait d'obtenir un alignement de la législation européenne sur la législation britannique, perçue comme une sorte de minimum garanti, en obtenant par exemple le vote d'un European Race Relations Act, au lieu d'un alignement sur le plus petit commun dénominateur aux différents Etats membres.

La constatation de l'absence quasi-totale de toute référene à la "race" dans les documents émanant des instances européennes paraît alors d'autantplus inquiétante à ces Britanniques blacks.

Il est évident que le processus d'intégration européenne les met dans une situation particulièrement inconfortable. Comme le faisait remarquer Valérie Amos, de l'Equal Opportunity Commission: "Il est profondément ironique de constater que la Commission for Racial Equality (CRE) et l'Equal Opportunity Commission (EOC) soient maintenant considérées comme des modèles de ce qui devrait exister plus largement en Europe. Notre position est alors essentiellement défensive, (et consiste) à nous raccrocher à tout gain obtenu" 20 . Ceux-là même qui se battent depuis des années en Grande-Bretagne contre l'insuffisance des lois contre la discrimination raciale

19. Le terme "immigrant" est en effet souvent considéré comme péjoratif en GrandeBretagne ; dès lors, certains estiment que ce n'est pas parce que les "Blacks" du continent n'ont pas réussi à se défaire de cette étiquette infamante pour que ceux d'Outre-Manche se la voient appliquée.

20. SCORE (UK), 24/11/90, paragraphe 4.8. 
devraient maintenant, face à la situation prévalant sur le continent, s'estimer satisfaits de celles-ci, voire même s'ériger en leurs défenseurs.

Racisme et citoyenneté

La question soulevée est alors de manière plus fondamentale celle des rapports entre citoyenneté et racisme.

Ainsi en France, on a longtemps estimé, et on estime encore, que l'égalité de tous est assurée par l'idéal républicain de l'égalité essentielle de chaque individu, telle qu'inscrite dans la Déclaration des Droits de l'Homme et du citoyen ; et que ces principes sont non seulement suffisants, mais les seuls pertinents, pour assurer l'égalité réelle. Dès lors, c'est l'accès à la citoyenneté, et donc à la nationalité, dans l'état actuel des choses, qui est conçu comme source principale de cette égalité.

Le débat porte alors sur la contradiction entre le primat du droit et la persistance de discriminations, y compris et/ou surtout dans les situations d'égalité formelle. Les Britanniques blacks ont donc raison lorsqu'ils attirent l'attention sur le danger latent de l'intégration européenne telle qu'elle serait conçue aujourd'hui : obliger ceux qui sont citoyens communautaires à se contenter de cette "qualité", à être reconnaissants d'être citoyens, sans chercher à en dépasser les limites évidentes. Cette logique viendrait en effet renforcer les conceptions de la citoyenneté comme un privilège, dont il faudrait être reconnaissant d'être les récipiendiaires sans demander plus.

La question ouverte est alors celle d'un éventuel équilibre à trouver entre d'une part le primat du droit formel, de la citoyenneté comme catégorie suffisante pour structurer les droits dans la nouvelle configuration des sociétés européennes, et d'autre part l'enjeu essentiel d'un dispositif permettant l'émergence d'une égalité réelle entre les citoyens.

Dans le cas français, le principe fondateur de l'égalité de tous les citoyens-individus ne permet pas, dans la conception actuelle, l'adoption de politiques du type discriminations positives pour certaines catégories de citoyens ; plus encore l'existence même d'intérêts collectifs pouvant se représenter ou être représentés, est dans l'idéal déniée ${ }^{21}$.

En Grande-Bretagne, une telle notion d'égalité essentielle entre tous les citoyens, ou sujets, n'a jamais réellement existé. Des statuts ont pu et peuvent encore se chevaucher par lesquels des sujets ont accès à plus de droits que d'autres ; c'est ainsi que les sujets originaires du Commonwealth et n'ayant pas la nationalité britannique jouissent des "privilèges civiques" sur le territoire britannique, mais n'ont pas droit au regroupement familial ou à la libre circulation en Europe.

21. Sur ce sujet, voir entre autres C. Neveu, 1993, ainsi que N. Rouland, 1993 
Plus encore, le discours sur "l'identité nationale" y est très largement racialisé, ce qui d'une part tend à exclure encore plus les Blacks de la communauté imaginée de ceux qui "appartiennent à la nation", et d'autre part légitime ou en tout cas explique l'émergence de formes d'organisation axées sur la "race" et la lutte contre les discriminations.

L'existence d'une telle contradiction entre l'insuffisance du seul accès à la citoyenneté pour garantir l'égalité réelle et la mise en avant de la seule lutte contre la discrimination au détriment de la citoyenneté ouvre alors sur une alternative.

On peut tout d'abord insister sur la citoyenneté comme étant un statut normal, minimal, allant de soi et devant être complété par la mise en oeuvre de politiques antidiscriminatoires, voire de politiques de discrimination positive. L'accent est alors mis sur la lutte contre le racisme, et la citoyenneté devient une question secondaire. C'est la tendance qui dominerait dans les organisations de Britanniques blacks.

On peut certes également s'accrocher à une vision immobiliste de la citoyenneté "à la française" en la déclarant encore pleinement adaptée aux nouvelles conditions, ce qui ne va pas sans risque à plus ou moins long terme.

Une troisième voie consisterait à mettre l'accent sur la nécessité de reconsidérer le contenu même de la citoyenneté au regard de la situation actuelle. Ce dont il s'agit alors est de remettre en cause le mythe, particulièrement vivace en France, de l'homogénéité culturelle de la nation, de reconnaître la diversité des origines et des références, peutêtre y compris par la mise en oeuvre d'un certain type de "discrimination positive", tout en redonnant un sens dynamique à la citoyenneté. C'est ce qui a pu être proposé par la notion de "nouvelle citoyenneté" ; l'objectif est alors de redonner à cette citoyenneté son caractère actif, de dépasser une pratique largement passive de celle-ci, aujourd'hui pratiquement réduite au vote, ou à l'abstention. Cette redéfinition inclut alors l'ensemble des citoyens quelque soit leur origine, et propose que les termes concrets d'une égalité réelle soient réélaborés en commun, sans fragmentation de la collectivité des citoyens selon des lignes "ethniques" ou "raciales". Au-delà d'une citoyenneté strictement légale, exo-définie par l'Etat et imposée sur chaque individu, il s'agirait de mettre en avant une citoyenneté auto-définie, réappropriée de manière positive par tous.

C'est aussi au coeur de ces contradictions que des structures comme le Forum des Migrants ou SCORE (UK) évoluent. Le premier présente le risque de reproduire une vision en partie dépassée des populations issues de l'immigration en Europe, de les maintenir, voire de les renvoyer à une extranéité qui ne correspond plus totalement à la réalité. Il pourrait ainsi maintenir une perception de ces populations comme "corps étrangers" aux sociétés européennes, appelés un jour ou l'autre à en repartir. Plus encore, en ne traitant qu'implicitement des questions de racisme, le Forum des Migrants introduit une division au sein des populations immigrées, en fonction du statut. 
SCORE (UK) se heurte aux mêmes problèmes lorsque, dans ses tentatives de rentrer en contact avec ceux qui en France sont victimes de discrimination, il se refuse à admettre la moindre validité de la notion de "black" comme catégorie politique significative ; cette structure court alors le risque, en estimant que le refus d'une grande partie des populations issues de l'immigration en France de se désigner comme black relève de la pure aliénation, de voir se dissiper toute possibilité de collaboration dynamique entre les pays européens.

La confusion des catégories dans ces deux structures montre d'ailleurs clairement la complexité des questions soulevées : est-ce le statut qui fournit aujourd'hui le principe de différenciation central dans l'accès aux droits et aux ressources; ou est-ce que, comme le disait l'un des participants à la conférence inaugurale de SCORE (UK) "la couleur de votre passeport est moins importante que la couleur de votre visage" ?

Il va de soi que tant que l'image de l'Europe restera celle d'une Europe "blanche", cette affirmation restera valide. C'est donc bien non seulement le racisme qu'il faut combattre, mais aussi les cristallisations des conceptions des différentes identités nationales comme "racialement" homogènes. Dans l'idée quasi-hégémonique aujourd'hui en Europe de la coextension Etat/nation, la seule citoyenneté ne peut suffire faire évoluer ces perceptions, sauf s'il y a redéfinition collective des critères de la citoyenneté,actifs plus que passifs, issus de droits acquis ou encore à acquérir, plus que de privilèges innés et hérédiraires, ou encore fixés de manière anhistorique. Ainsi, de même que "black" a pu à une époque être réapproprié positivement par ceux qui étaient ainsi désignés, ne peuton penser comme possible la réappropriation par les citoyens eux-mêmes de la citoyenneté, d'une redéfinition active de l'appartenance à la politie ? Du même coup, une partie au moins des tensions entre racisme et citoyenneté, entre égalité formelle et égalité réelle, pourraient être résolues.

\section{Références bibliographiques :}

- DE JONG, Deny, et ZWAMBORN, Marcel ; 1991. Equal treatment and discrimination in Europe, Final report of the fesability study on co-operation of organisations in Europe concerned with improving the position of migrants and ethnic minorities, Commandé par International Alert et The Netherlands Institute of Human rights en vue de la Conférence 1991 "Action on Racism in Europe", avril.

- NEVEU, Catherine ; 1993. Communauté, nationalité et citoyenneté, Paris, Karthala.

- NEVEU, Catherine ; 1993. "Minority formation and national identity : France and Britain", Communication au Séminaire scientifique Ethnicity, Nationalism and Culture in Western Europe (The facelift of Europe), Université d'Amsterdam, 24-27 février. 
- Race and Class ; 1991. "Europe : variations on a theme of racism", Volume 32, n 3, janvier-mars.

- ROULAND, Norbert ; 1993. "La France s'interroge sur a meilleure manière d'intégrer les étrangers", Le Monde diplomatique, octobre.

- SCORE (UK) ; 1990. Compte-rendu de la Conférence inaugurale de SCORE (UK), Birmingham, 24/11/90. 